OPEN ACCESS

Edited by:

Guy A. Rutter,

Imperial College London,

United Kingdom

Reviewed by:

Marcia Hiriart,

Universidad Nacional

Autonoma de Mexico, Mexico

Guoqiang Gu,

Vanderbilt University,

United States

${ }^{*}$ Correspondence:

A. Dean Sherry

dean.sherry@utsouthwestern.edu

${ }^{t}$ These authors have contributed equally to this work

Specialty section:

This article was submitted to Diabetes: Molecular Mechanisms, a section of the journal Frontiers in Endocrinology

Received: 14 December 2020 Accepted: 26 April 2021

Published: 26 May 2021

Citation:

Clavijo Jordan V, Hines CDG, Gantert $L T$, Wang $S$, Conarello $S$,

Preihs $C$, Chirayil S, Klimas $M$,

Evelhoch $\mathrm{J} L$ and Sherry $A D$ (2021) Imaging Beta-Cell Function

in the Pancreas of Non-Human

Primates Using a Zinc-Sensitive MRI Contrast Agent.

Front. Endocrinol. 12:641722. doi: 10.3389/fendo.2021.641722

\section{Imaging Beta-Cell Function in the Pancreas of Non-Human Primates Using a Zinc-Sensitive MRI Contrast Agent}

\author{
Veronica Clavijo Jordan ${ }^{1,2 \dagger}$, Catherine D. G. Hines ${ }^{3 \dagger}$, Liza T. Gantert ${ }^{3}$, Shubing Wang ${ }^{4}$, \\ Stacey Conarello ${ }^{5}$, Christian Preihs ${ }^{2,6}$, Sara Chirayil ${ }^{2}$, Michael Klimas ${ }^{3}$, \\ Jeffrey L. Evelhoch ${ }^{3}$ and A. Dean Sherry ${ }^{2,6,7,8^{*}}$ \\ ${ }^{1}$ Athinoula A. Martinos Center for Biomedical Imaging, Massachusetts General Hospital, Harvard Medical School, \\ Charlestown, MA, United States, ${ }^{2}$ Advanced Imaging Research Center, The University of Texas Southwestern Medical \\ Center, Dallas, TX, United States, ${ }^{3}$ Translational Biomarkers, Merck \& Co., Inc., Kenilworth, NJ, United States, ${ }^{4}$ Biometrics \\ Research, Merck \& Co., Inc., Kenilworth, NJ, United States, ${ }^{5}$ Pharmacology, Merck \& Co., Inc., Kenilworth, NJ, \\ United States, ${ }^{6}$ VitalQuan, LLC, Dallas, TX, United States, ${ }^{7}$ Department of Radiology, The University of Texas Southwestern \\ Medical Center, Dallas, TX, United States, ${ }^{8}$ Department of Chemistry \& Biochemistry, The University of Texas at Dallas, \\ Richardson, TX, United States
}

Non-invasive beta cell function measurements may provide valuable information for improving diabetes diagnostics and disease management as the integrity and function of pancreatic beta cells have been found to be compromised in Type- 1 and Type-2 diabetes. Currently, available diabetes assays either lack functional information or spatial identification of beta cells. In this work, we introduce a method to assess the function of beta cells in the non-human primate pancreas non-invasively with MRI using a Gd-based zinc(II) sensor as a contrast agent, Gd-CP027. Additionally, we highlight the role of zinc(II) ions in the paracrine signaling of the endocrine pancreas via serological measurements of insulin and c-peptide. Non-human primates underwent MRI exams with simultaneous blood sampling during a Graded Glucose Infusion (GGl) with Gd-CP027 or with a nonzinc(II) sensitive contrast agent, gadofosveset. Contrast enhancement of the pancreas resulting from co-release of zinc(II) ion with insulin was observed focally when using the zinc(II)-specific agent, Gd-CP027, whereas little enhancement was detected when using gadofosveset. The contrast enhancement detected by Gd-CP027 increased in parallel with an increased dose of infused glucose. Serological measurements of C-peptide and insulin indicate that Gd-CP027, a high affinity zinc(II) contrast agent, potentiates their secretion only as a function of glucose stimulation. Taken in concert, this assay offers the possibility of detecting beta cell function in vivo non-invasively with MRI and underscores the role of zinc(II) in endocrine glucose metabolism.

Keywords: beta cell function, imaging, primate, diabetes, pancreas 


\section{INTRODUCTION}

Glucose metabolism is tightly controlled by a systemic feedbackloop mechanism comprised of communication between beta cells in the pancreas and insulin-sensitive tissues (1). The disturbance of glucose homeostasis as a result of immunologically-induced loss of pancreatic beta cells ultimately leads to symptoms of type 1 diabetes mellitus (T1DM). This disease usually presents itself in young individuals, and common treatment options include self-injected doses of long-acting and short-acting insulin derivatives, mechanical monitoring of glucose and insulin dosing via pumps, and pancreatic islet transplantation $(2,3)$. In contrast, Type 2 diabetes mellitus (T2DM), responsible for approximately $90 \%$ of all diabetes cases (4), represents a systemic disease characterized by hyperglycemia either in the context of insulin resistance in peripheral tissues or lack of insulin secretion from the pancreas $(1,5)$. The treatment paradigm for T2DM includes initial lifestyle changes to attempt to reduce insulin resistance often supplemented with oral metformin, a biguanide-class drug whose primary mechanism of action is to reduce hepatic glucose production while simultaneously increasing insulin uptake in insulin-sensitive tissues (6). Although effective at reducing hyperglycemia, metformin has not been proven to prevent the conversion from impaired glucose tolerance to frank T2DM, only slowing the progression of disease while maintaining the rate of beta cell function deterioration when compared to other treatment options such as sulfonylureas or insulin injections (7). The pathophysiology of the disease is vastly heterogeneous across patients and this creates uncertainties in the optimal treatment regimen (1). While therapeutic outcomes may be predicted by knowing the state of beta-cell functionality (8), at this time there is no accurate way to measure beta-cell function directly from the pancreas in a noninvasive fashion.

Beta cell function is currently assessed by measuring blood glucose, proinsulin, insulin and C-peptide levels, or clamping experiments (9-12). However, blood levels lack information on the volume of beta cells (beta cell mass) or recruitment of cells (beta cell dynamics) needed to maintain blood glucose levels. While beta cell mass loss is well-known to parallel pancreatic function (13), it does not provide a complete picture of beta cell failure that is also attributable to beta cell dysfunction (14). Spatial or dynamic beta cell function information could be helpful to investigate the relationship of beta cell function to mass loss, where the latter can be measured invasively $(15,16)$ or non-invasively $(17,18)$. Both beta cell mass and function deficits contribute to T2DM (13) although some data suggests that beta cell function is more relevant and elusive in this disease $(13,14)$, particularly when beta cells are present but not viable (19). Thus, the means to non-invasively and longitudinally measure beta-cell function would be highly advantageous to determine the time course of T2DM development and for monitoring interventions to preserve beta-cell function.

Magnetic Resonance Imaging (MRI) is routinely used in abdominal diagnoses, particularly those related to the structural integrity of the pancreas. This includes ductal anatomy, presence of cysts, or acute pancreatitis, and diagnosis of lesions (20). Standard extracellular contrast agents are routinely used in abdominal MRI exams for tumor identification and staging. However, most if not all, currently conducted pancreatic radiological exams offer no direct information about beta cell mass or function. Zinc(II) is known to be co-released with insulin from beta cells in response to an increase in blood glucose. This ion is then dispersed into the islet extracellular space where it is taken up by neighboring alpha cells serving as an inter-cellular messenger ion signaling for glucagon secretion (21). The secreted zinc(II) can then be used as an indirect indicator of insulin release (i.e., beta cell function) by use of a zinc(II) responsive contrast agent. A number of MRI zinc(II)-responsive agents have been reported (22-25) with designs largely based upon formation of a ternary complex with serum albumin in the presence of excess zinc(II). Upon co-release of zinc(II) and insulin from pancreatic betacells, these agents form a complex with the excess zinc(II) and subsequently bind with albumin to form a high-relaxivity complex that ultimately translates into hyperintense voxels in a $\mathrm{T}_{1}$-weighted MRI image. Studies in rodents have demonstrated that this allows functional mapping of the insulin profile in vivo in healthy and diabetic rodents by MRI $(22,26,27)$. However, it is generally appreciated that the rodent pancreas is an amorphous organ dispersed along the abdomen and interlaced between the intestines, stomach, liver, and spleen so it is exceedingly difficult to identify the endocrine lobes both accurately and consistently to monitor beta-cell function or loss in a longitudinal manner as a consequence of diabetes. As a result, a larger animal model in which the pancreas is a solid organ with easily identifiable lobes is paramount to further evaluate the use of such agents for functional imaging of glucose-stimulated insulin secretion.

A technique for imaging beta-cell function directly from different regions of the pancreas or other tissues could be quite useful for islet transplantation studies. Identification of dysfunctional islets in the pancreas along with accurate sorting of xenograft islets would most certainly benefit from an imaging method designed to assess beta-cell function in order to maximize the chances of graft success. In the treatment of T2DM, it would be beneficial to obtain beta-cell functional information to understand the pathogenesis of T2DM, treatment response and ultimately aid in the selection of improved T2DM therapies. In this work, we describe the use of the previously reported zinc(II) sensor (GdCP027) $(22,28)$ to measure beta-cell function in healthy nonhuman primates. In order to evaluate the sensitivity and specificity of this assay, we perform a modified graded glucose infusion (GGI) paradigm to initiate zinc(II)/insulin co-release in the presence of Gd-CP027 versus a control agent (gadofosveset) with similar albumin binding behavior and relaxivity but lacking in sensitivity to zinc(II).

\section{MATERIALS AND METHODS}

\section{Animal Preparation}

All procedures were performed in accordance with our institution's IACUC guidelines at the AALAC-accredited facility where the animals were housed. Adult, healthy male 
and female ( $\mathrm{n}=2$, each) rhesus macaques were used for this study; alternative large animal models (e.g. swine, canines) were not used because primates allow for easier translation to human studies. All four animals were imaged on two separate days, Study 1 and Study 2, which were approximately three weeks apart to allow for adequate recovery after anesthesia and blood sampling. Animals underwent a 16 hour fast prior to the day of the MRI exam, and water intake was restricted two hours prior to anesthesia to facilitate easier visualization of the pancreas.

The animals were fed a High Protein Monkey Diet 5045 and High Protein Monkey Diet Jumbo 5047 biscuits once a day, where daily intake was calculated and tailored to each animal as a function of age, weight, and body condition. The animals weighed $7.1 \pm 1.1 \mathrm{~kg}$ (range, 6.3-9.7 kg) on both study days. To facilitate handling in preparation for MRI, animals were first anesthetized with $10 \mathrm{mg} / \mathrm{kg}$ ketamine hydrochloride $(100 \mathrm{mg} /$ $\mathrm{mL}$ ) administered intramuscularly so intravenous catheters could be placed into the left and right saphenous veins and a cephalic vein for contrast agent, glucose infusion, and blood sampling, respectively. Animals were then administered $5 \mathrm{mg} / \mathrm{kg}$ of propofol intravenously via the cephalic vein to allow endotracheal intubation. Once placed on the scanning table, anesthesia was maintained using a mix of approximately 70:30 ratio of oxygen to isoflurane, which was then maintained between $1.5-3 \%$. A temperature probe, pulse oximeter and end tidal $\mathrm{CO}_{2}$ monitor were connected for continuous monitoring. Body temperature was maintained by a dorsal K-module warm water recirculating blanket. Fluid maintenance $(10 \mathrm{~mL} / \mathrm{kg} / \mathrm{hr}$ Lactated Ringer's solution) was co-administered in conjunction with the GGI platform during the MRI exam, as described in main text.

Breath-hold imaging was also performed on the anesthetized animals during the dynamic 3D T1-weighted images only. Animals were pre-selected based on their ability to breath-hold during previous MRI exams. The gas lines to the ventilator were shut off when breath-holding was needed and was then opened five seconds before breath-holding needed to end. All breathholds were less than 40 seconds, and at least three minutes were allowed for recovery between breath-holds, consistent with our animal protocol. Each animal was able to receive enough oxygen and remain physiologically stable while under anesthesia. $\mathrm{SpO}_{2}$ was monitored for any irregularities. $\mathrm{SpO}_{2}$ did not drop lower than 95 , which would require longer recovery between breathholds and adjustment of anesthesia, if necessary. Adjustments to the amount of air administered, ratio of oxygen to isoflurane, and percent of isoflurane were carried out as needed to maintain stable anesthesia and breath-holding under the supervision of a veterinarian.

\section{MRI Acquisition}

All imaging was performed using a Siemens 3T Trio and a fourchannel flex coil. After a series of localizer images to position the region of the abdomen where the T1-weighted images would be acquired, T2-weighted axial and coronal single-shot turbo spin echo images were acquired with respiratory triggering to identify the pancreas. Using these spin echo images, higher resolution, fat-saturated T2-weighted turbo spin echo images of the pancreas were acquired with respiratory triggering in preparation for the T1-weighted 3D gradient echo dynamic sequence. For dynamic imaging, an axial, breath-held $3 \mathrm{D} \mathrm{T}_{1^{-}}$ weighted ("T1W") sequence was acquired with the following parameters: FOV $=20 \mathrm{~cm} \times 20 \mathrm{~cm}$, slice thickness $=1.0 \mathrm{~mm}$, TE/ $\mathrm{TR}=2.32 / 5.75 \mathrm{~ms}, 1$ average, 30 slices, parallel acceleration factor $=2$, flip-angle $=10^{\circ}, 192 \times 192$ matrix for a resolution of $1.0 \mathrm{~mm} \times 1.0 \mathrm{~mm} \times 1.0 \mathrm{~mm}, \mathrm{BW}=360 \mathrm{~Hz} /$ pixel, and a scan time of 25 seconds.

In Study 1, the animals were administered $50 \mathrm{mg} / \mathrm{kg} / \mathrm{hr} \mathrm{Gd}$ CP027 intravenously. For comparison purposes, this amounts to $\sim 2-3$ fold less contrast agent that that typically administered as a single bolus $(0.1 \mathrm{mmol} / \mathrm{kg})$ in a clinical study. Lyophilized, research grade Gd-CP027 (VitalQuan, Dallas, TX) was reconstituted in $100 \mathrm{mM}$ TRIS buffer, $\mathrm{pH} 7.40(25.5 \mathrm{mg} / \mathrm{mL})$ and filtered for sterility. In Study 2, gadofosveset trisodium was administered intravenously at $50 \mathrm{mg} / \mathrm{kg} / \mathrm{hr}$. Commercially available gadofosveset was diluted into $100 \mathrm{mM}$ TRIS buffer, $\mathrm{pH} 7.40(25.2 \mathrm{mg} / \mathrm{mL})$ and filtered for sterility. The two imaging studies were performed at least three weeks apart.

Infusion of contrast agent was initiated after all anatomical images of the pancreas and an initial T1W image was acquired. Immediately after initiating the infusion, the first dynamic T1W, breath-held image was acquired (“-20 min”). The infusion was then continued for the entirety of the exam. After 20 minutes of waiting to ensure the contrast agent was well-distributed and in steady-state, dynamic T1-weighted images were acquired beginning at " $0 \mathrm{~min}$ " over one hour (“60 $\mathrm{min}$ ") at four-minute intervals (17 total time points). The 20 min pre-circulation time was established by imaging the kidneys every five minutes after infusion until constant enhancement was seen in the renal pelvis (data not shown).

\section{Graded Glucose Infusion and Blood Sampling}

To challenge the pancreas to co-release insulin and zinc(II), a modified 2-step graded glucose infusion (GGI) protocol, a standard paradigm for pancreas challenges (29-31), was performed during the MRI exam. Intermittent blood samples were also collected during the study for glucose, insulin, and Cpeptide measurements. Figure 1 summarizes the simultaneous MRI acquisition, blood sampling, and infusion paradigms. The GGI consisted of $20 \mathrm{~min}$ of saline infusion $(0 \mathrm{~min}-20 \mathrm{~min})$ as an internal control, followed by 20 minutes of $8 \mathrm{mg} / \mathrm{kg} / \mathrm{min}$ dextrose (20 $\mathrm{min}-40 \mathrm{~min}$ ), and then 20 minutes of $16 \mathrm{mg} / \mathrm{kg} / \mathrm{min}$ dextrose (40 min - $60 \mathrm{~min}$ ). Blood samples were taken every four minutes in EDTA tubes beginning at 0 min and continuing for one hour $(60 \mathrm{~min})$ at $4 \mathrm{~min}$ intervals.

\section{MRI Analysis}

All images were analyzed using both VivoQuant (v2.50, inviCRO, LLC, Boston, MA) and ImageJ (National Institutes of Health, Bethesda, MD). The pancreas was manually subdivided into body/head/neck and tail regions of interest (ROIs), with the plane containing the superior pole of the left kidney identified as the division between the body/head/neck and tail. Small circular or ellipsoid-shaped hyperintense regions, typically 15-20 pixels 

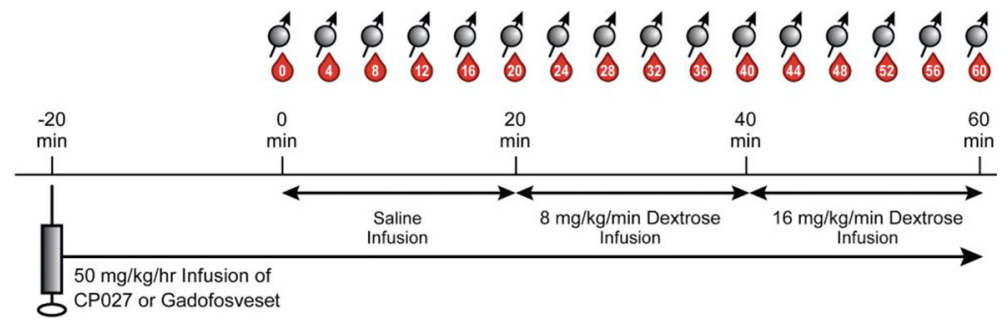

FIGURE 1 | Altered GGl paradigm. After animals were anesthetized and anatomical images were acquired, $50 \mathrm{mg} / \mathrm{kg} / \mathrm{hr}$ of contrast agent was infused intravenously. After waiting $20 \mathrm{~min}$ for the agent to circulate, a separate saline infusion was administered for $20 \mathrm{~min}$ ( 0 min - 20 min), followed by 20 min of 8 mg/ $\mathrm{kg} / \mathrm{min}$ dextrose (20 min - $40 \mathrm{~min}$ ), and then $20 \mathrm{~min}$ of $16 \mathrm{mg} / \mathrm{kg} / \mathrm{min}$ of dextrose (40 min - $60 \mathrm{~min}$ ). Blood sampling (denoted as red drops) and breath-held T1W images (spin symbols) were acquired every $4 \mathrm{~min}$.

in size, were observed predominantly in the pancreatic tail region during infusion of Gd-CP027 $(n \geq 4)$ and recorded. These regions are hereafter referred to as "hotspots". During infusion of gadofosveset, a largely albumin-bound vascular agent, fewer and less intense hotspots were also detected but these likely reflect small vascular regions of increased blood flow stimulated by insulin secretion $(32,33)$. To compare the signal intensities of these hotspots with nearby regions of lower intensity, $4-7$ similarly sized and shaped ROIs on the same imaging slice adjacent to the identified hotspots were also measured in images collected during infusion of either Gd-CP027 of gadofosveset. These ROIs are hereafter referred to as "withinslice comparator"). The signal intensity at each time point in all ROIs in the pancreas (whole pancreas, tail, body/head/neck, hotspots, within-slice comparators) was normalized to the signal intensity in nearby muscle ROIs at the same time points. Using the normalized signal intensities in the pancreas ROIs, the percent change from 0 min (initiation of saline infusion; hereafter referred to as "\% Signal Change") was calculated for each ROI at each time point.

Additionally, hotspots were categorized and clustered by size (area in $\mathrm{mm}^{2}$ ) using the "Grow region" tool on Horos dicom viewing software. The pancreas prior to contrast agent infusion was segmented and the average and standard deviation in signal intensity of a $5 \mathrm{~mm}$-thick slab was measured. The smart ROI selection tool was programmed to only select regions of interest that were 2-standard deviations above the average segmented pre-infusion pancreas signal intensity. Every imaging time point was analyzed in this manner and the ROIs were identified by average signal intensity, standard deviation, and size. This sequence was repeated for all animals in both studies. A Kmeans clustering algorithm was performed in order to determine hotspot clusters by size and change in contrast-to-noise ratio as a function of blood c-peptide, where the centroid of the clusters was identified by minimizing the sum of the squared distance between the centroid and each data point.

\section{Blood Analysis}

Plasma samples were analyzed for glucose, insulin, and Cpeptide levels at each time point (In Vitro Sciences Laboratory, David H. Murdock Research Institute, Kannapolis, NC).
Immediately after collection, blood was centrifuged at $1300 \mathrm{~g}$ at $4^{\circ} \mathrm{C}$ for 10 minutes. The plasma was removed, and frozen at $80^{\circ} \mathrm{C}$ for analysis. Glucose levels $(\mathrm{mg} / \mathrm{dL})$ were obtained using an Analox Glucose Analyzer (Analox Instruments Ltd, UK) from plasma samples and a commercially available glucose kit. Insulin levels $(\mu \mathrm{IU} / \mathrm{mL})$ and C-peptide levels $(\mathrm{ng} / \mathrm{mL})$ were obtained using a Meso Scale Discovery Sector Imager S 600 (Meso Scale Diagnostics, LLC, Rockville, MD) with commercially available insulin and C-peptide custom kits, respectively.

\section{Statistical Analysis}

A sequential statistical analysis was performed using two models (34). The first model estimated the slope of each parameter vs. time for each contrast agent to characterize agent and time interaction ("slope analysis") for C-peptide, glucose, insulin, and \%signal change in the whole pancreas, head/body/neck, tail, hotspots, and comparator ROIs. Whenever the slope analysis identified differences between the two agents, a second analysis was performed. This second model used the means of each time point for each parameter and each agent to determine the time at which the two agents diverge ("time course analysis"). To avoid inflating false positives, rigorous multiplicity adjustments were performed and resulted in splitting the $\mathrm{p}$-value between the slope analysis and the time course analysis. A p-value of $0.025(=0.05 /$ 2) was used to determine statistical significance; a Bonferroni Correction for the multiple time point comparisons $(n=15)$ in the time course analysis was applied using an adjusted significance level $\sim 0.00167(=0.05 / 2 / 15)$ to control the familywise error rate at the 0.025 level.

\section{RESULTS}

Figure 2A depicts the rhesus pancreas in vivo and plane of MRI acquisition shown in Figure 2B. Figure 2B shows representative $\mathrm{T}_{1}$-weighted MRI images acquired at the 16,36 , and 56-min time points corresponding to the end of the three different infusion blocks (i.e. saline, $8 \mathrm{mg} / \mathrm{kg} / \mathrm{min}$ dextrose, and $16 \mathrm{mg} / \mathrm{kg} / \mathrm{min}$ dextrose). In study 1 , steady-state contrast enhancement was detected in the abdominal aorta and inferior vena cava by the end of the first infusion block (saline, 20 mins). Upon initiation of 
dextrose infusion at $8 \mathrm{mg} / \mathrm{kg} / \mathrm{min}$, the image intensity of the pancreas was further enhanced and small regions of higher intensity "hot spots" were detected primarily in the tail region. Signal enhancement throughout the pancreas and the hotspots became even more prominent when the dextrose level was increased to $16 \mathrm{mg} / \mathrm{kg} / \mathrm{min}$. The bottom panel of Figure 2B (study 2) shows images of the pancreas of the same animal and the same time points as in Study 1 after infusion of gadofosveset. Here, less enhancement was evident in images of the pancreas either before (16 min image) or after infusion of glucose (images at 36 and $56 \mathrm{~min}$ ). Figure $\mathbf{2 C}$ compares the changes in image enhancement over the entire time course within the regions identified as hotspots after infusion of Gd-CP027 plus glucose versus gadofosveset plus glucose. All animals fully recovered from anesthesia after each study.

The average image intensity within ROIs consisting of the entire pancreas, the tail alone, and body/head/neck alone for the 4 animals are compared in Figures 3A, B. The increase in MR signal intensity over time in study 1 versus study 2 did not differ significantly for any of the segmented regions but an analysis of the slopes of these curves indicate there are statistically significant differences for the whole pancreas segment versus the body/head/neck region ( $\mathrm{p}=5.29 \mathrm{E}-7$ and $2.79 \mathrm{E}-6$, respectively).
Like the comparator ROI data shown in Figure 2C, the segmented whole pancreas and body/head/neck time course data did not diverge significantly at any point during the GGI ( $p \geq 0.00167$ for all). The slope analysis for the tail region showed no differences between agents $(\mathrm{p}=0.92)$.

Figure 3 show plots of image intensity increase $( \pm S D, n=4)$ for the segmented pancreas (Figures 3B-D) and for within-slice comparator ROIs (Figure 3E) and hotspot ROIs (Figure 3F) over the 60 min study. The hotspot ROIs showed a 3\% increase in normalized signal intensity during infusion gadofosveset versus an $8 \%$ increase during infusion of Gd-CP027 over the first $16 \mathrm{~min}$. These differences likely reflect differences in the extracellular distribution of gadofosveset (largely vascular) versus Gd-CP027 (both vascular and extracellular). However, after initiation of the GGI, these differences in ROI image intensity diverged even further with the gadofosveset data remaining near $5 \%$ at $60 \mathrm{~min}$ versus near $20 \%$ for Gd-CP027. These differences can only be attributed to responsiveness of GdCP027 to $\mathrm{Zn}$ (II) release in these well-defined regions of the pancreas. An analysis of slopes of these curves also reported significant differences [hotspot ROIs ( $\mathrm{p}=1.81 \mathrm{E}-12)$ versus comparator ROIs $(\mathrm{p}=0.003)]$. The hotspot data comparisons between the two studies diverged significantly at minute 44
A
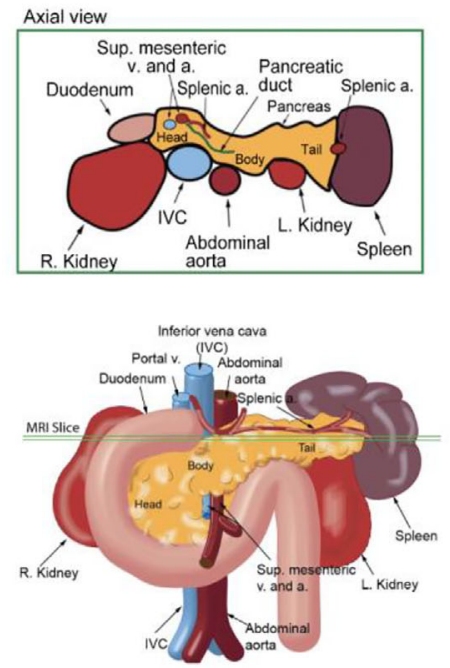

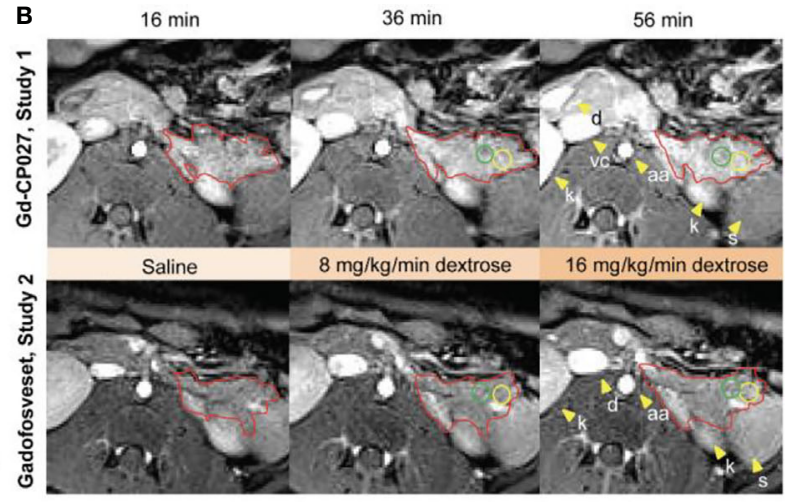

C

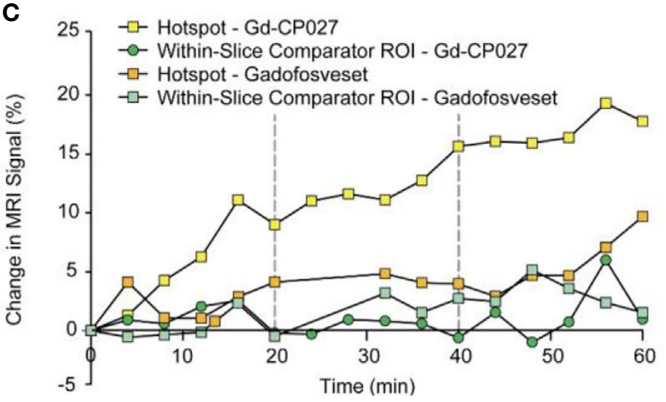

FIGURE 2 | (A) Schematic illustrating non-human primate abdominal anatomy, including organs surrounding the pancreas, and an axial cross-section indicating imaging slice used for MRI pancreas localization and quantification. (B) In vivo MR imaging of non-human primate after infusion of Gd-CP027 (Study 1), and gadofosveset (Study 2). Both studies received the altered $\mathrm{GGl}$, and 3D $\mathrm{T}_{1}$-weighted images were collected every four minutes. Axial images show the $\mathrm{T}_{1}$-weighted scans at 16, 36, and $56 \mathrm{~min}$ time points, where each represent the scan after complete saline infusion, $8 \mathrm{mg} / \mathrm{kg} / \mathrm{min}$ dextrose, and $16 \mathrm{mg} / \mathrm{kg} / \mathrm{min}$ dextrose infusion, respectively. The tail of the pancreas is highlighted in both panels (red) and Gd-CP027 shows a more prominent enhancement in the pancreas, with focal signal enhancements intensifying over time. Hotsposts (yellow) and adjacent comparator ROls (green) were identified and measured, and are plotted in (C) for the animal displayed in (B) 
A

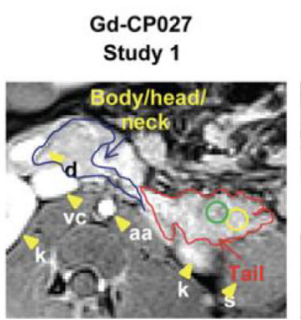

C

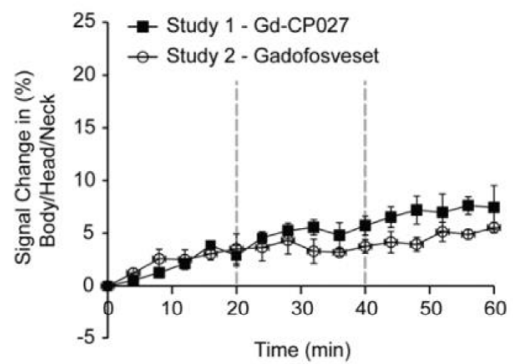

E

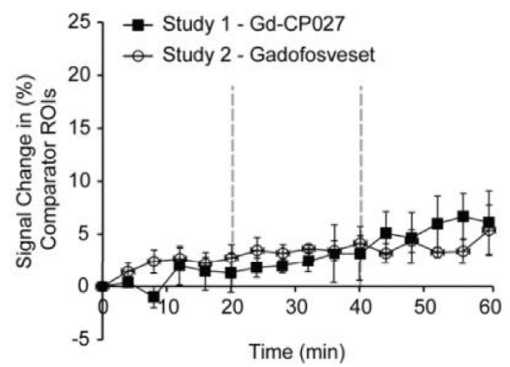

B

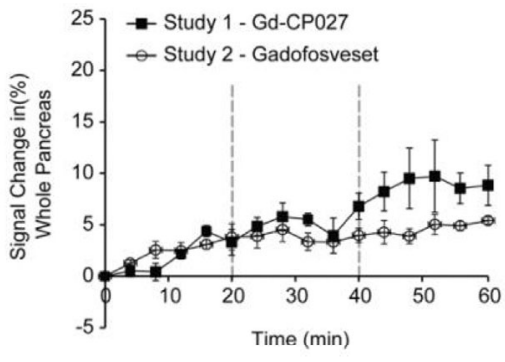

D

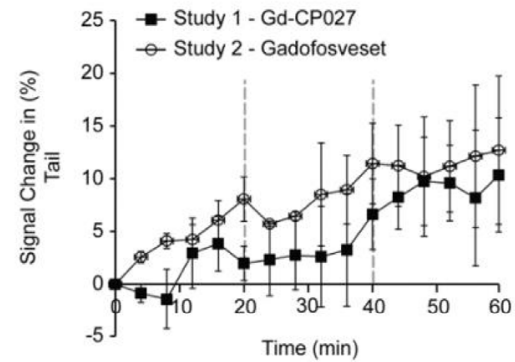

F

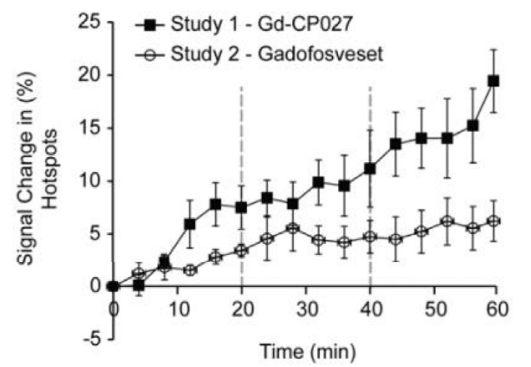

FIGURE 3 | (A) Non-human pancreas segmentation delineating the tail (red outline), body/head/neck (blue outline) and ROls within the tail. Change in signal intensity (\%) after infusion of each agent alone (0-20 min) and after graded increases in glucose (20-40 and 40-60 min). for (B) the entire pancreas, (C) Body/Head/Neck, and (D) Tail of the pancreas. The error bars reflect standard deviations for the 4 animals. (E) Change in MRI signal intensity (\%) for within-slice comparator ROIs for the 4 animals. (F) Change in MRI signal intensity (\%) within the regions identified as hotspots in the 4 animals.

(after infusion of glucose at the higher level) and remained divergent through the end of the GGI. The comparator ROI time courses did not diverge significantly at any time point during the GGI.

The blood measurements showed the expected increase in blood glucose during each dextrose infusion period in both studies (Figure 4A). An analysis of slopes of these curves showed no significant differences between study groups $(\mathrm{p}=0.07)$. As expected, insulin and C-peptide increased in parallel with plasma glucose (Figures 4B, C) although, interestingly, both insulin and C-peptide were consistently higher in the Gd-CP027 study after infusion of glucose in comparison to the gadofosveset study. The slopes of these timedependent increases were significantly different for both insulin $(\mathrm{p}=$ 4.64E-5) and C-peptide ( $\mathrm{p}=3.29 \mathrm{E}-5)$. These differences indicate that Gd-CP027 enhances insulin secretion above that initiated by glucose. By analyzing the area under the curve of the hotspot signal for both Gd-CP027 and Gadofosveset we observe that only GdCP027 corelates tightly to the blood measurements, in particular to insulin and C-peptide (Figures 4D-F).

To examine whether there are differences in the hotspot signal intensity and size patterns with respect to C-peptide levels in blood, plots of dCNR (increase in CNR compared to an equallysized ROI in muscle) versus plasma C-peptide are shown in Figures 5A, B. Hotspots of various sizes were identified and included in this plot only if the average intensity was 2-standard deviations higher than the pre-infusion signal intensity. Further clustering of hotspot dCNR was done by using an algorithm to minimize the distance between the centroid of the clusters and each data point (K-means clustering). The same number of clusters $(\mathrm{k}=4)$ was used in both plots. The average hotspot size in the Gd-CP027 group ranged from $12-50 \mathrm{~mm}^{2}$ in comparison to an average size of $22-52 \mathrm{~mm}^{2}$ in the gadofosveset group. One animal displayed hyper-physiological secretion of C-peptide/insulin in response to glucose as shown by the light blue colored cluster of $12 \mathrm{~mm}^{2}$ in sized hotspots in Figure 5A. This illustrates that not all animals release the same amount of C-peptide when stimulated by a similar increase in plasma glucose. Nonetheless, this K-means cluster analysis showed a positive correlation between hotspot dCNR versus blood C-peptide levels in the Gd-CP027 group but not in the gadofosveset group. 


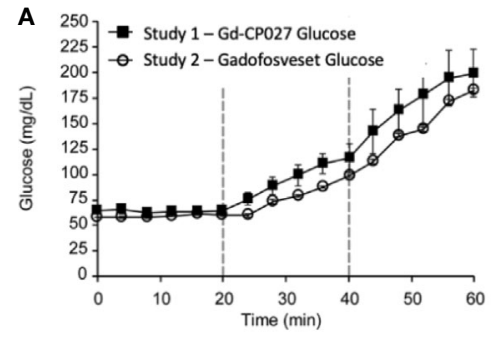

D
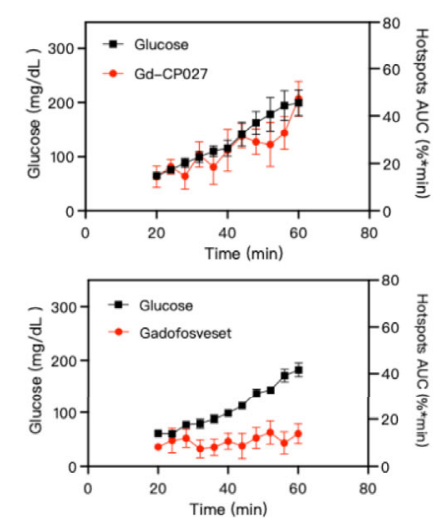

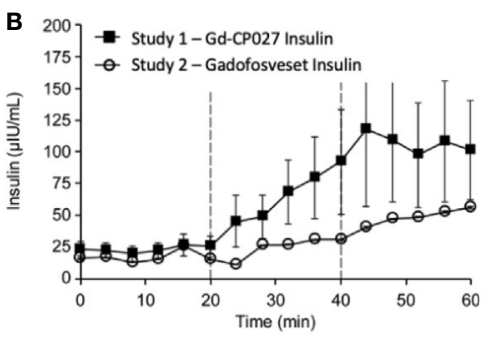

E
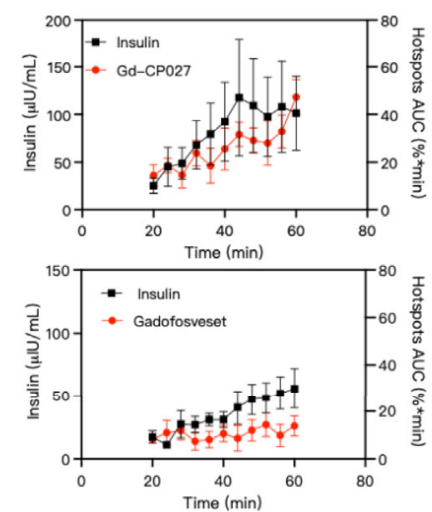

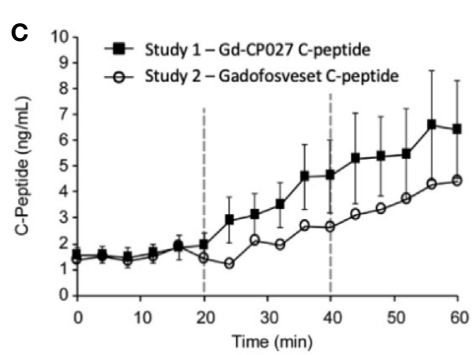

$\mathbf{F}$
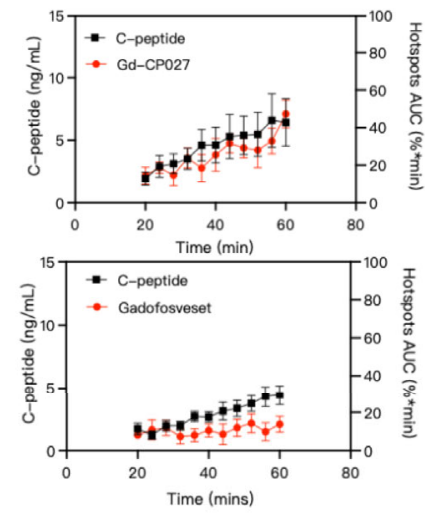

FIGURE 4 | (A) Blood glucose, (B) insulin, and (C) C-peptide levels collected during each GGl study $(n=4)$. Error bars represent standard error, and vertical dashed lines reflect each infusion period. Area under the curve for Gd-CP027 and Gadofosveset hotspots overlapped to blood measurements of (D) Glucose, (E) Insulin, and (F) C-peptide.

\section{DISCUSSION}

As demonstrated previously in isolated islets and in the rodent pancreas $(26,27)$, this imaging study of the non-human primate pancreas demonstrates that it may be feasible to assess $\beta$-cell function locally in vivo by glucose-stimulated zinc secretion (GSZS) and a Gd-based zinc(II) sensor as a contrast agent. Given that GSZS could also reflect $\beta$-cell mass, given the spatial resolution of MRI and the inability to resolve individual $\beta$-cells in this study it is deemed that this technology more accurately reflect $\beta$-cell function. Although MR signal enhancement was detected throughout the pancreas upon a graded glucose infusion (GGI) in the presence of either Gd-CP027 (zinc-sensitive agent) or gadofosveset (zinc insensitive), small regions previously referred to as "hotspots" (27) did show significant differences between the two agents during the highest GGI infusion period (44-60 min, Figure 3F). The blood plasma panel (Figures 4A-C) showed that glucose homeostasis was not significantly disturbed by infusion of either contrast agent (prior to initiation of the first glucose infusion) yet the insulin and C-peptide profiles suggest that Gd-CP027 may potentiate insulin secretion, especially at the highest level of glucose infusion. Since insulin secretion and potentiation by Gd-CP027 occurred only after infusion of glucose, the potentiation appears to be related to $\operatorname{zinc}(\mathrm{II})$ released in response to glucose and not the
A

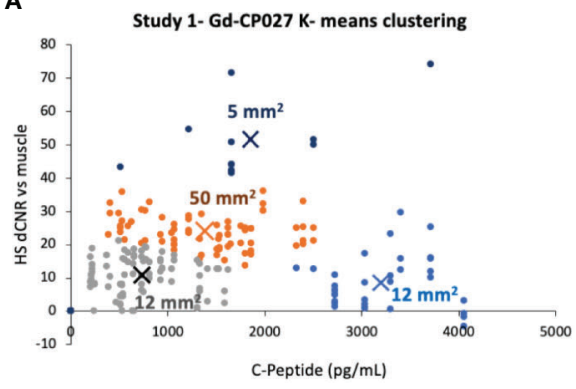

B

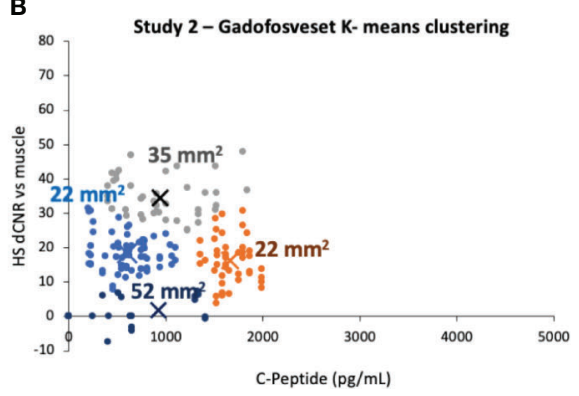

FIGURE 5 | K-means clustering ( $\mathrm{k}=4$ for both studies) showing the change in contrast-to-noise ratio versus the corresponding blood C-peptide measurement for all animals and all hotspots, where "X" represents the centroid of each cluster labeled with its respective average hotspot area for animals receiving (A) Gd-CP027, or (B) Gadofosveset. 
initial extracellular zinc(II) present in tissue. These observations are further confirmed in Figures 4D-F where the area under the curve (AUC) for the hotspots over the 20 - 60 minute infusion periods corelate tightly with insulin and c-peptide plasma profiles over time. However, further experiments will be necessary to understand the mechanism of Gd-CP027 potentiation, but it is important to note that neither Gd-CP027 nor gadofosveset are thought to enter cells so this potentiation likely reflects a disturbance in extracellular zinc (II) levels.

It is well known that zinc(II) plays a role in the correct function of many secretory organs including prostate, mammary glands, and the pancreas $(21,35-37)$. In the pancreas, cosecretion of zinc and insulin has been imaged using fluorescent probes $(38,39)$ and more recently by MRI $(22,24,26)$. Given that MRI sensors detect insulin secretion indirectly by binding to excess $\mathrm{Zn}$ (II) released with insulin, it is important to consider the potential impact these agents have on other pancreatic secretory cell mechanisms. Secretory cells have distinct mechanisms to maintain tightly controlled $\mathrm{Zn}$ (II) homeostasis. This is mainly achieved by the expression of $\mathrm{Zn}$ (II) transporters of the $\mathrm{ZnT}$ and ZIP families (36). It has been shown that extracellular signals, such as exposure of prostate epithelial cells to an increase in plasma glucose (40), triggers a rapid redistribution of $\mathrm{Zn}$ (II) transporters between the plasma membrane and other cellular organelle membranes that results in also rapid redistribution of mobile $\mathrm{Zn}$ (II). Many other studies in multiple cell types have shown that alterations in various extracellular processes induces an immediate redistribution of intracellular $\mathrm{Zn}$ (II), a phenomenon referred to as "the zinc wave" (41). These and other studies showing that $\mathrm{Zn}$ (II) mimics the actions of hormones, growth factors, and cytokines have led to the concept that $\mathrm{Zn}$ (II) ions play a role as a second messenger capable of transducing extracellular stimuli into intracellular signaling pathways (42). Several different $\mathrm{Zn}$ (II) transporters are expressed in $\beta$-cells but the most highly abundant transporter, ZnT8, playing a key role in formation of zinc-insulin granules (43) and ZnT8 expression has been shown to positively correlate with circulating levels of insulin and glucagon (44). Although controversial, release of $\mathrm{Zn}$ (II) and insulin from $\beta$ cells has been reported to modulate glucagon secretion by uptake of $\mathrm{Zn}$ (II) ions into neighboring $\alpha$-cells via ZIP transporters (21). Given that Gd-CP027 has a high affinity for $\mathrm{Zn}(\mathrm{II})\left(\mathrm{K}_{\mathrm{D}} \approx 30 \mathrm{nM}\right)$, infusion of this agent certainly must have an influence on the activity of $\mathrm{Zn}(\mathrm{II})$ transporters and likely could impact signaling of glucagon secretion $(36,45,46)$. Although further studies will be required to determine the exact mechanism of insulin potentiation by $\mathrm{Zn}$ (II) chelating agents, we hypothesize that the origin of the observed potentiation of glucose-dependent insulin secretion by Gd-CP027 may reflect $\mathrm{Zn}$ (II) scavenging and subsequent calciummediated cell-membrane depolarization $(21,47)$. Although the plasma glucose levels tended to be higher in animals infused with Gd-CP027 versus gadofosveset (Figure 4A), these differences did not reach statistical significance. Nevertheless, this trend would be consistent with the hypothesis that a lower concentration of bioavailable $\mathrm{Zn}$ (II) in the extracellular space in animals exposed to Gd-CP027 could allow unabated glucagon secretion from $\alpha$-cells.
One of the most interesting findings in this study was the focal nature of the normalized image enhancement in select regions of the pancreas. These focal hotspots, observed mostly in the tail, are consistent with clusters of islets as reported in previously published studies $(27,32,33,48)$. It is known that the regional distribution of pancreatic islets is uneven and clustered throughout the pancreas (32) and that this distribution varies among animal species $(49,50)$. The non-human primate, like the human pancreas, has insulinsecreting islets dispersed throughout the body of the pancreas, with higher $\beta$-cell density in the tail (50). Based on these prior reports, we expected to find significant differences in the segmented whole tail. An analysis of size and signal change clustering of hotspots (Figure 5A) showed that the smallest hotspots correlated with higher levels of C-peptide in blood even though there were no significant differences in image intensity in the entire segmented tail because of averaging with non-enhancing pancreas regions.

Although we observed that the change in signal intensity of hotspot clusters in the pancreas correlated with insulin and Cpeptide secretion only in the Gd-CP027 group (Figures 4D-F), it was interesting to find observable hotspots in the pancreas in the gadofosveset group as well. These results indicate that these focal enhancements do not only reflect insulin and $\mathrm{Zn}$ (II) secretion but there must be an additional mechanism that contributes to these higher intensity regions in the gadofosveset study. Given that gadofosveset is a blood pool agent that extravasates into extracellular space more slowly than other low molecular weight Gd-based agents, it is reasonable to suggest that the focal enhancements observed in the gadofosveset study may reflect an increase in blood flow in those regions releasing the most insulin. It has been shown previously using fluorescence-labeled red blood cells that the apparent blood volume of the pancreas is greater during hyperglycemia than during hypoglycemia $(51,52)$ and this may be the origin of the effects observed here when using the vascular agent, gadofosveset.

The primary limitation of this study was the small number of animals available for study. Given the small cohort and the fact that one animal released more insulin and C-peptide while generating smaller changes in CNR in the Gd-CP027 study compared to the other animals, the differences between many of the parameters measured in this study did not reach statistical significance. Another potential limitation could be differences in pharmacokinetics and biodistribution of the two contrast agents. It was evident that uptake/clearance rates may differ, as seen in the 0-20 minute interval of Figure 2C and in the kidneys of Figure $\mathbf{2 B}$, so the tissue biodistribution could contribute to local concentration differences at any given time point during the study. An analysis of slopes in the time-dependent data revealed significant differences between contrast agents for all measured parameters except the tail and glucose levels while the time course analysis revealed divergent responses at specific times only for the hotspot data. However, a consistent trend in the time course was evident in the plots (Figures 3 and 4) demonstrating different C-peptide and insulin release, as well as increased enhancement with Gd-CP027. This discrepancy between the sequential tests is due to a very conservative multiplicity adjustment as well as low power for the time course analysis due to the small sample size. Despite these limitations, this first study in the 
non-human primate pancreas offers the possibility of imaging $\beta$-cell function in vivo $(38,47,53)$.

\section{DATA AVAILABILITY STATEMENT}

The original contributions presented in the study are included in the article/supplementary material. Further inquiries can be directed to the corresponding author.

\section{ETHICS STATEMENT}

The animal study was reviewed and approved by the Institutional Animal Care and Use Committee at Merck \& Co., Inc.

\section{REFERENCES}

1. Kahn SE, Cooper ME, Del Prato S. Pathophysiology and Treatment of Type 2 Diabetes: Perspectives on the Past, Present, and Future. Lancet (2014) 383 (9922):1068-83. doi: 10.1016/S0140-6736(13)62154-6

2. Bruni A, Gala-Lopez B, Pepper AR, Abualhassan NS, Shapiro AJ. Islet Cell Transplantation for the Treatment of Type 1 Diabetes: Recent Advances and Future Challenges. Diabetes Metab Syndr Obes (2014) 7:211-23. doi: 10.2147/ DMSO.S50789

3. Atkinson MA, Eisenbarth GS, Michels AW. Type 1 Diabetes. Lancet (2014) 383(9911):69-82. doi: 10.1016/S0140-6736(13)60591-7

4. Shi Y, Hu FB. The Global Implications of Diabetes and Cancer. Lancet (2014) 383(9933):1947-8. doi: 10.1016/S0140-6736(14)60886-2

5. Melmed S, Polonsky K, Larsen PR, Kronenberg H. Williams Textbook of Endocrinology. 12. Philadelphia, PA USA: Elsevier/Saunders (2011).

6. White JRJr. A Brief History of the Development of Diabetes Medications. Diabetes Spectr (2014) 27(2):82-6. doi: 10.2337/diaspect.27.2.82

7. Page KA, Reisman T. Interventions to Preserve Beta-Cell Function in the Management and Prevention of Type 2 Diabetes. Curr Diabetes Rep (2013) 13 (2):252-60. doi: 10.1007/s11892-013-0363-2

8. Nguyen KT, Billington CJ, Vella A, Wang Q, Ahmed L, Bantle JP, et al. Preserved Insulin Secretory Capacity and Weight Loss Are the Predominant Predictors of Glycemic Control in Patients With Type 2 Diabetes Randomized to Roux-en-Y Gastric Bypass. Diabetes (2015) 64(9):3104-10. doi: $10.2337 / \mathrm{db} 14-1870$

9. Lee SW, Lee S, Kim SH, Kim TH, Kang BS, Yoo SH, et al. Parameters Measuring Beta-Cell Function are Only Valuable in Diabetic Subjects With Low Body Mass Index, High Blood Glucose Level, or Long-Standing Diabetes. Yonsei Med J (2011) 52(6):939-47. doi: 10.3349/ymj.2011.52.6.939

10. U.K. Prospective Diabetes Study 16. Overview of 6 Years' Therapy of Type II Diabetes: A Progressive Disease. U.K. Prospective Diabetes Study Group. Diabetes (1995) 44(11):1249-58. doi: 10.2337/diabetes.44.11.1249

11. Festa A, Williams K, D'Agostino RJr., Wagenknecht LE, Haffner SM. The Natural Course of Beta-Cell Function in Nondiabetic and Diabetic Individuals: The Insulin Resistance Atherosclerosis Study. Diabetes (2006) 55(4):1114-20. doi: 10.2337/diabetes.55.04.06.db05-1100

12. Sjaarda L, Lee S, Tfayli H, Bacha F, Bertolet M, Arslanian S. Measuring BetaCell Function Relative to Insulin Sensitivity in Youth: Does the Hyperglycemic Clamp Suffice? Diabetes Care (2013) 36(6):1607-12. doi: 10.2337/dc12-1508

13. Meier JJ, Bonadonna RC. Role of Reduced Beta-Cell Mass Versus Impaired Beta-Cell Function in the Pathogenesis of Type 2 Diabetes. Diabetes Care (2013) 36 Suppl 2:S113-9. doi: 10.2337/dcS13-2008

14. Leahy JL, Hirsch IB, Peterson KA, Schneider D. Targeting Beta-Cell Function Early in the Course of Therapy for Type 2 Diabetes Mellitus. J Clin Endocrinol Metab (2010) 95(9):4206-16. doi: 10.1210/jc.2010-0668

15. Reiner T, Thurber G, Gaglia J, Vinegoni C, Liew CW, Upadhyay R, et al. Accurate Measurement of Pancreatic Islet Beta-Cell Mass Using a Second-

\section{AUTHOR CONTRIBUTIONS}

VCJ, AS, MK, JE, CH, and SCo conceived of the project. CH, LG, SW, VCJ, and JE carried out the experiments and analyzed the data. $\mathrm{CP}, \mathrm{SCh}$, and AS designed and prepared the zinc-sensitive imaging agent. All authors contributed to the article and approved the submitted version.

\section{FUNDING}

This work was supported in part by grants to ADS from the National Institutes of Health (DK-095416) and the American Diabetes Association (7-12-IN-42).
Generation Fluorescent Exendin-4 Analog. Proc Natl Acad Sci USA (2011) 108 (31):12815-20. doi: 10.1073/pnas.1109859108

16. Rahier J, Guiot Y, Goebbels RM, Sempoux C, Henquin JC. Pancreatic BetaCell Mass in European Subjects With Type 2 Diabetes. Diabetes Obes Metab (2008) 10 Suppl 4:32-42. doi: 10.1111/j.1463-1326.2008.00969.x

17. Blomberg BA, Codreanu I, Cheng G, Werner TJ, Alavi A. Beta-Cell Imaging: Call for Evidence-Based and Scientific Approach. Mol Imaging Biol (2013) 15 (2):123-30. doi: 10.1007/s11307-013-0620-4

18. Jodal A, Schibli R, Behe M. Targets and Probes for non-Invasive Imaging of Beta-Cells. Eur J Nucl Med Mol Imaging (2016) 44(4):712-27. doi: 10.1007/ s00259-016-3592-1

19. Gotthardt M, Eizirik DL, Cnop M, Brom M. Beta Cell Imaging - a Key Tool in Optimized Diabetes Prevention and Treatment. Trends Endocrinol Metab (2014) 25(8):375-7. doi: 10.1016/j.tem.2014.02.002

20. Zamboni G, Gourtsoyianni S. MDCT and MRI of Liver, Bile Ducts and Pancreas. Italy: Springer (2015). p. 121. doi: 10.1007/978-88-470-5720-3_26

21. Kelleher SL, McCormick NH, Velasquez V, Lopez V. Zinc in Specialized Secretory Tissues: Roles in the Pancreas, Prostate, and Mammary Gland. Adv Nutr (2011) 2(2):101-11. doi: 10.3945/an.110.000232

22. Yu J, Martins AF, Preihs C, Clavijo Jordan V, Chirayil S, Zhao P, et al. Amplifying the Sensitivity of Zinc(II) Responsive MRI Contrast Agents by Altering Water Exchange Rates. J Am Chem Soc (2015) 137(44):14173-9. doi: 10.1021/jacs.5b09158

23. De Leon-Rodriguez LM, Lubag AJ, Lopez JA, Andreu-de-Riquer G, AlvaradoMonzon JC, Sherry AD. A Second Generation MRI Contrast Agent for Imaging Zinc Ions In Vivo. Medchemcomm (2012) 3(4):480-3. doi: 10.1039/c2md00301e

24. Esqueda AC, Lopez JA, Andreu-de-Riquer G, Alvarado-Monzon JC, Ratnakar J, Lubag AJ, et al. A New Gadolinium-Based MRI Zinc Sensor. J Am Chem Soc (2009) 131(32):11387-91. doi: 10.1021/ja901875v

25. Major JL, Parigi G, Luchinat C, Meade TJ. The Synthesis and In Vitro Testing of a Zinc-Activated MRI Contrast Agent. Proc Natl Acad Sci USA (2007) 104 (35):13881-6. doi: 10.1073/pnas.0706247104

26. Lubag AJ, De Leon-Rodriguez LM, Burgess SC, Sherry AD. Noninvasive MRI of Beta-Cell Function Using a Zn2+-responsive Contrast Agent. Proc Natl Acad Sci USA (2011) 108(45):18400-5. doi: 10.1073/pnas.1109649108

27. Martins AF, Clavijo Jordan V, Bochner F, Chirayil S, Paranawithana N, Zhang $\mathrm{S}$, et al. Imaging Insulin Secretion From Mouse Pancreas by MRI is Improved by Use of a Zinc-Responsive Mri Sensor With Lower Affinity for $\mathrm{Zn}(2+)$ Ions. J Am Chem Soc (2018) 140(50):17456-64. doi: 10.1021/jacs.8b07607

28. Clavijo Jordan MV, Lo ST, Chen S, Preihs C, Chirayil S, Zhang S, et al. ZincSensitive MRI Contrast Agent Detects Differential Release of Zn(II) Ions From the Healthy vs. Malignant Mouse Prostate. Proc Natl Acad Sci USA (2016) 113(37):E5464-71. doi: 10.1073/pnas.1609450113

29. Wang X, Hansen BC, Shi D, Fang Y, Du F, Wang B, et al. Quantification of Beta-Cell Insulin Secretory Function Using a Graded Glucose Infusion With C-peptide Deconvolution in Dysmetabolic, and Diabetic Cynomolgus Monkeys. Diabetol Metab Syndr (2013) 5(1):40. doi: 10.1186/1758-5996-5-40 
30. Lin JD, Wu CZ, Tang WH, Kuo FC, Pei D, Liang YJ, et al. Comparison of Second-Phase Insulin Secretion Derived From Standard and Modified LowDose Graded Glucose Infusion Tests. Can J Diabetes (2016) 40(6):529-34. doi: 10.1016/j.jcjd.2016.03.007

31. Kim SH, Liu A, Ariel D, Abbasi F, Lamendola C, Grove K, et al. Effect of Salsalate on Insulin Action, Secretion, and Clearance in Nondiabetic, InsulinResistant Individuals: A Randomized, Placebo-Controlled Study. Diabetes Care (2014) 37(7):1944-50. doi: 10.2337/dc13-2977

32. Ionescu-Tirgoviste C, Gagniuc PA, Gubceac E, Mardare L, Popescu I, Dima S, et al. A 3D Map of the Islet Routes Throughout the Healthy Human Pancreas. Sci Rep (2015) 5:14634. doi: 10.1038/srep14634

33. Zhu S, Larkin D, Lu S, Inouye C, Haataja L, Anjum A, et al. Monitoring CPeptide Storage and Secretion in Islet Beta-Cells In Vitro and In Vivo. Diabetes (2016) 65(3):699-709. doi: 10.2337/db15-1264

34. Pinheiro JC, Bates DM. Mixed-Effects Models in S and s-PLUS. New York: Springer (2004).

35. Liu Y, Batchuluun B, Ho L, Zhu D, Prentice KJ, Bhattacharjee A, et al. Characterization of Zinc Influx Transporters (Zips) in Pancreatic Beta Cells: Roles In Regulating Cytosolic Zinc Homeostasis and Insulin Secretion. J Biol Chem (2015) 290(30):18757-69. doi: 10.1074/jbc.M115.640524

36. Chimienti F. Zinc, Pancreatic Islet Cell Function and Diabetes: New Insights Into an Old Story. Nutr Res Rev (2013) 26(1):1-11. doi: 10.1017/S0954422412000212

37. Haase H, Rink L. Functional Significance of Zinc-Related Signaling Pathways in Immune Cells. Annu Rev Nutr (2009) 29:133-52. doi: 10.1146/annurevnutr-080508-141119

38. Li D, Chen S, Bellomo EA, Tarasov AI, Kaut C, Rutter GA, et al. Imaging Dynamic Insulin Release Using a Fluorescent Zinc Indicator for Monitoring Induced Exocytotic Release (ZIMIR). Proc Natl Acad Sci USA (2011) 108 (52):21063-8. doi: 10.1073/pnas.1109773109

39. Qian WJ, Aspinwall CA, Battiste MA, Kennedy RT. Detection of Secretion From Single Pancreatic Beta-Cells Using Extracellular Fluorogenic Reactions and Confocal Fluorescence Microscopy. Anal Chem (2000) 72(4):711-7. doi: $10.1021 /$ ac $991085 \mathrm{t}$

40. Lo ST, Parrott D, Jordan MVC, Joseph DB, Strand D, Lo UG, et al. The Roles of ZnT1 and ZnT4 in Glucose-Stimulated Zinc Secretion in Prostate Epithelial Cells. Mol Imaging Biol (2021) 23(2):230-40. doi: 10.1007/s11307-020-01557-x

41. Yamasaki S, Sakata-Sogawa K, Hasegawa A, Suzuki T, Kabu K, Sato E, et al. Zinc is a Novel Intracellular Second Messenger. J Cell Biol (2007) 177(4):63745. doi: $10.1083 / \mathrm{jcb} .200702081$

42. Beyersmann D, Haase H. Functions of Zinc in Signaling, Proliferation and Differentiation of Mammalian Cells. Biometals (2001) 14(3-4):331-41. doi: 10.1023/A:1012905406548

43. Lemaire K, Chimienti F, Schuit F. Zinc Transporters and Their Role in the Pancreatic Beta-Cell. J Diabetes Investig (2012) 3(3):202-11. doi: 10.1111/ j.2040-1124.2012.00199.x

44. Cauchi S, Del Guerra S, Choquet H, D’Aleo V, Groves CJ, Lupi R, et al. MetaAnalysis and Functional Effects of the SLC30A8 rs13266634 Polymorphism on Isolated Human Pancreatic Islets. Mol Genet Metab (2010) 100(1):77-82. doi: 10.1016/j.ymgme.2010.01.001

45. Ravier MA, Rutter GA. Glucose or Insulin, But Not Zinc Ions, Inhibit Glucagon Secretion From Mouse Pancreatic Alpha-Cells. Diabetes (2005) 54(6):1789-97. doi: 10.2337/diabetes.54.6.1789

46. Franklin I, Gromada J, Gjinovci A, Theander S, Wollheim CB. Beta-Cell Secretory Products Activate Alpha-Cell ATP-dependent Potassium Channels to Inhibit Glucagon Release. Diabetes (2005) 54(6):1808-15. doi: 10.2337/diabetes.54.6.1808

47. MacDonald PE, Rorsman P. Oscillations, Intercellular Coupling, and Insulin Secretion in Pancreatic Beta Cells. PLoS Biol (2006) 4(2):e49. doi: 10.1371/ journal.pbio.0040049

48. Stefan Y, Meda P, Neufeld M, Orci L. Stimulation of Insulin Secretion Reveals Heterogeneity of Pancreatic B Cells In Vivo. J Clin Invest (1987) 80(1):175-83. doi: 10.1172/JCI113045

49. Wang X, Misawa R, Zielinski MC, Cowen P, Jo J, Periwal V, et al. Regional Differences in Islet Distribution in the Human Pancreas-Preferential BetaCell Loss in the Head Region in Patients With Type 2 Diabetes. PLoS One (2013) 8(6):e67454. doi: 10.1371/journal.pone.0067454

50. Kim A, Miller K, Jo J, Kilimnik G, Wojcik P, Hara M. Islet Architecture: A Comparative Study. Islets (2009) 1(2):129-36. doi: 10.4161/isl.1.2.9480

51. Nyman LR, Ford E, Powers AC, Piston DW. Glucose-Dependent Blood Flow Dynamics in Murine Pancreatic Islets In Vivo. Am J Physiol Endocrinol Metab (2010) 298(4):E807-14. doi: 10.1152/ajpendo.00715.2009

52. Carlbom L, Espes D, Lubberink M, Eriksson O, Johansson L, Jansson L, et al. Pancreatic Perfusion and Subsequent Response to Glucose in Healthy Individuals and Patients With Type 1 Diabetes. Diabetologia (2016) 59 (9):1968-72. doi: 10.1007/s00125-016-4016-2

53. MacDonald PE, Braun M, Galvanovskis J, Rorsman P. Release of Small Transmitters Through Kiss-and-Run Fusion Pores in Rat Pancreatic Beta Cells. Cell Metab (2006) 4(4):283-90. doi: 10.1016/j.cmet.2006.08.011

Conflict of Interest: CH, LG, SW, SC, MK, and JE were employed by Merck \& Co., Inc. CP, one of the inventors of Gd-CP027, was employed by VitalQuan, LLC during the study. VitalQuan LLC licensed Gd-CP027 from the University of Texas and supplied the compound to Merck \& Co., Inc.

The remaining authors declare that the research was conducted in the absence of any commercial or financial relationships that could be construed as potential conflict of interest.

Copyright (c) 2021 Clavijo Jordan, Hines, Gantert, Wang, Conarello, Preihs, Chirayil, Klimas, Evelhoch and Sherry. This is an open-access article distributed under the terms of the Creative Commons Attribution License (CC BY). The use, distribution or reproduction in other forums is permitted, provided the original author(s) and the copyright owner(s) are credited and that the original publication in this journal is cited, in accordance with accepted academic practice. No use, distribution or reproduction is permitted which does not comply with these terms. 